\title{
Approaching to an optimal value of rise time in n-well/p substrate photodiode by controlling depletion layer width
}

\author{
Emad Hameed Hussein \\ Dept. of Physics, College of Science, \\ Al-Mustansiryia University, Baghdad, Iraq \\ E-mail:emadh67@yahoo.com
}

\begin{abstract}
The relationship between response speed of a silicon n-well/p substrate photodiode and the depletion layer width has been investigated. Variation of both the junction capacitance and the series resistance of the photodiode with the depletion layer width have been analyzed. It is shown that the contribution of the time constant and the drift time in the rise time within the depletion layer can be decreased to an optimal value (less than 1ns) just for specific value of the depletion layer width and smaller value of the diffused junction area.
\end{abstract}

Keywords: silicon photodiode, n-well/p junction, p-n junction, capacitance junction, series resistance.

Manuscript received 05.05.09; accepted for publication 10.09.09; published online 30.11.09.

\section{Introduction}

With the advances in technology over the past few years, it has become possible to fabricate high speed optoelectronic devices such as photodiode. High speed photodiodes play an important role in optical communication [1] and measurement systems [2], and hence they attract attention of researchers [3-5]. Unfortunately, realistic photodiodes have finite speed of responsivity. So, for a fast response time, through the fabrication process of the photodiode, impurity concentration and operating voltage must be chosen to give a depletion layer width so that the junction capacitance can cause as possible as short time constant. While many applications of photodiodes depend on thinner depletion region, others need to maximize thickness of the depletion region [6]. However, the series resistance which is due to bulk and contact resistances, and is usually only a few ohms, has an active role in the photodiode responsivity. Almost, the contacts are decreased by different methods like ion implantation [7] or by using titanium silicide [8], etc. The depletion layer is no longer dominating the series resistance as long as the substrate width is much larger than its width and hence a small resistivity semiconductor and small diffused junction area are required for lowering the series resistance. Although there are different semiconductor materials, silicon are commonly used to fabricate $\mathrm{p}-\mathrm{n}$ photodiodes for visible and near infrared region [9-12], as it was chosen in this research.

\section{Experimental}

In $n$-well/p substrate photodiode the depletion region is always wider than that for $\mathrm{p}-\mathrm{n}$ junction photodiode, because concentration levels of the n-well and the psubstrate are relatively low. Anyhow, structure of photodiode is essentially similar to $\mathrm{p}-\mathrm{n}$ junction diode except it has a transparent window can receive light through it. The photodiode model adopted in this research is similar to the model proposed by Swe and Yeo [8]. It is a p-substrate silicon type with resistivity $\rho=7.5 \mathrm{Ohm} \cdot \mathrm{cm}$ and a phosphorus n-well mask was implanted, as shown in Fig. 1. Arsenic implant and $\mathrm{BF} 2+$ implant were used to form the $\mathrm{n}^{+}$and $\mathrm{p}^{+}$regions, respectively, which are used as the cathode and anode contacts, respectively. Hence, a p-n junction is formed between $\mathrm{p}$-substrate and n-well region.

\section{Results and discussion}

The depletion layer width at thermal equilibrium for a one sided abrupt is given as [13]

$W_{D}=\sqrt{\frac{2 \varepsilon_{S}}{q N}\left(V_{b}-V-\frac{2 K T}{q}\right)}$

where $\varepsilon_{S}$ is the relative permittivity of the semiconductor, $q$ is the electron charge, $K$ is the Boltzmann constant, $T$ is the absolute temperature, $V_{b}$ is the built-in-potential, $V$ is the applied voltage and $N$ is 
the impurity concentration $\left(N\right.$ is $N_{A}$, if $N_{A}>>N_{D}$ or vise versa where $N_{A}$ is the accepter concentration and $N_{D}$ is the donor concentration). For silicon model $\varepsilon_{S}=k_{S} \cdot \varepsilon_{0}$, where $\varepsilon_{0}$ is the permittivity of free space $(8.85 \mathrm{pF} / \mathrm{m})$ and $k_{S}$ is silicon dielectric constant (11.68). By substituting these values and electron charge $q$ in Eq. (1), it becomes

$W_{D}=\sqrt{\frac{12.921}{N}\left(V_{b}-V-\frac{2 K T}{q}\right)}(\mu \mathrm{m})$

and so we can plot the depletion layer width $\left(W_{D}\right)$ as a function of impurity concentration $(N)$ for values changes from $10^{14} \mathrm{~cm}^{-3}$ to $10^{18} \mathrm{~cm}^{-3}$ and for different values of $\left(V_{b}-V-2 K T / q\right)$ changes from $1 \mathrm{~V}$ to $10 \mathrm{~V}$, as shown in Fig. 2.

Capacitance of the depletion layer of the photodiode is given in terms of the depletion layer width as

$C_{D}=\frac{\varepsilon_{S} A}{W_{D}}=\frac{\varepsilon_{S} A}{W_{D}}$,

where $A$ is the diffused junction area. By substituting the value of $\varepsilon_{S}$ in Eq. (3), it becomes

$C_{D}=103.368 \frac{A}{W_{D}}(\mathrm{pF})$,

where $A$ is measured in $\mathrm{mm}^{2}$. From Fig. 2 the values of $W_{D}$ varies approximately from $0.03 \mu \mathrm{m}$ to $12 \mu \mathrm{m}$. Thus, plotting the depletion layer capacitance as a function of the depletion layer width for different values of $A$ which changes from $1 \mathrm{~mm}^{2}$ to $20 \mathrm{~mm}^{2}$ with increment of $1 \mathrm{~mm}^{2}$ gives the graph shown in Fig. 3.

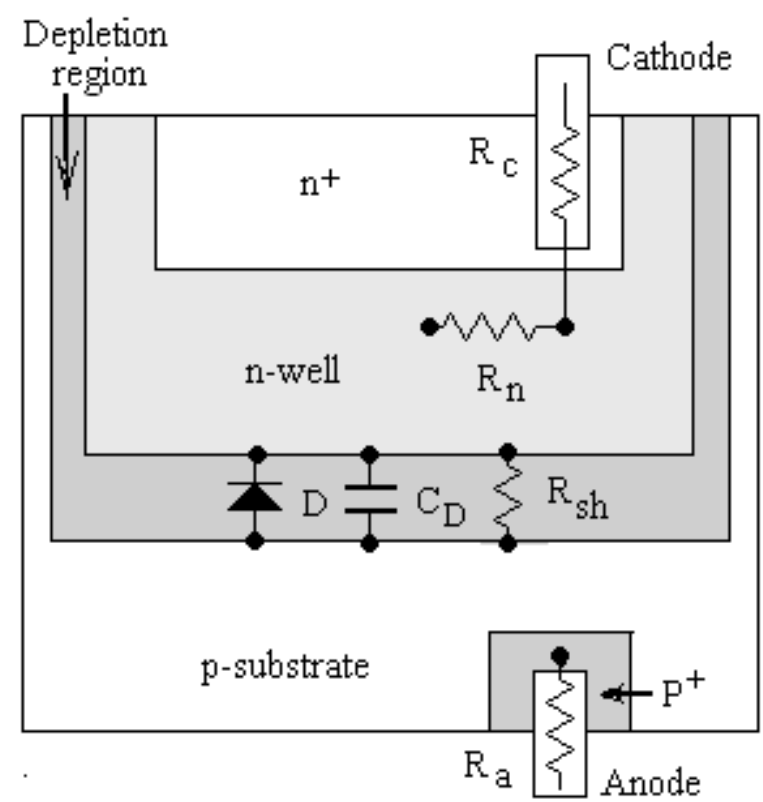

Fig. 1. Side view of the photodiode.

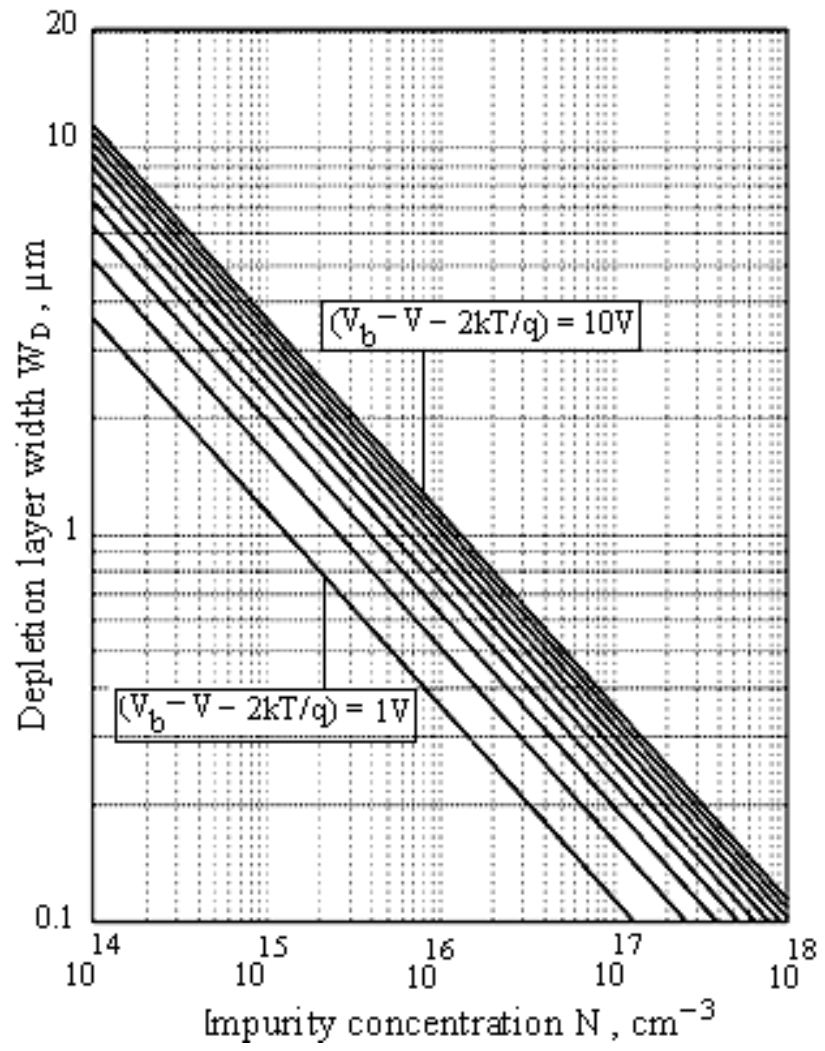

Fig. 2. The variation of the depletion layer width as a function of the impurity concentration at $300 \mathrm{~K}$.

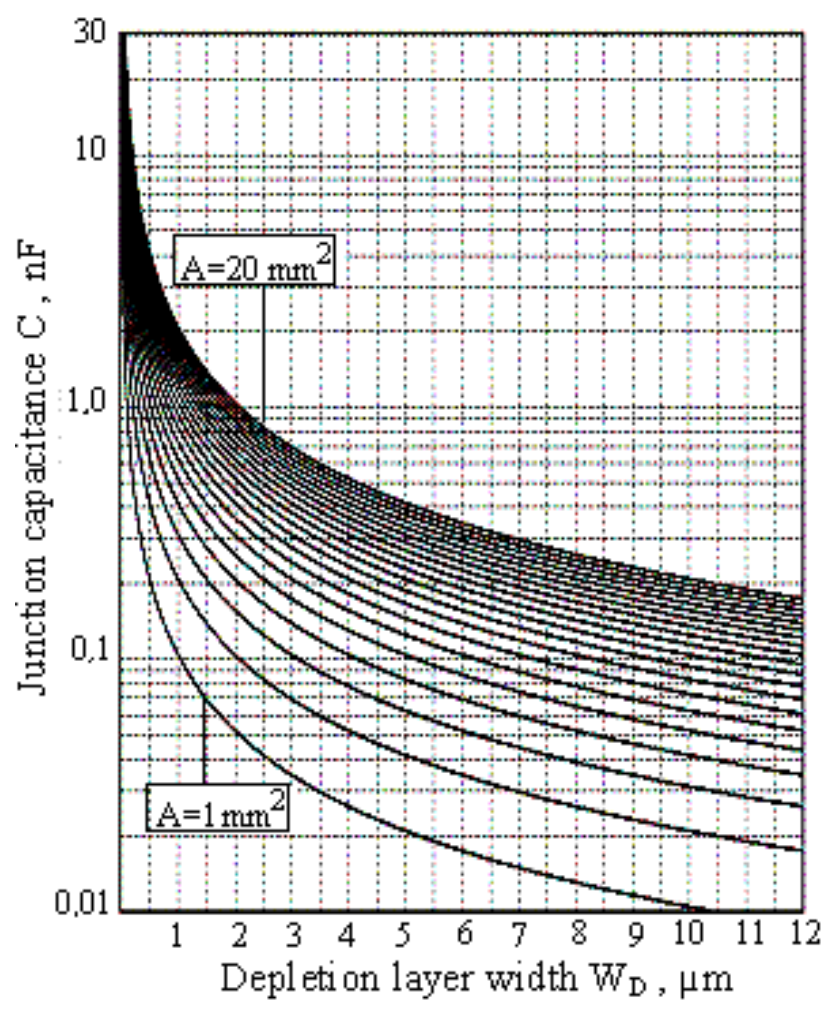

Fig. 3. The variation of the junction capacitance as a function of the depletion layer width at $300 \mathrm{~K}$. 
In $\mathrm{p}-\mathrm{n}$ photodiode the series resistance $R_{S}$ is considered an important factor of determining its response time, as it will be demonstrated later. The value of $R_{S}$ is given as

$$
R_{S}=\frac{\left(W_{S}-W_{D}\right) \rho}{A}+R_{n}+R_{C}
$$

where $W_{S}$ is the substrate thickness which was chosen to be $100 \mu \mathrm{m}, R_{n}$ is the resistance of the n-well region and $R_{C}$ is the contact resistances. As illustrated in Fig. $1 R_{C}$ is composed of $R_{a}$ and $R_{c}$; the anode and cathode resistances respectively, that is

$$
R_{C}=R_{a}+R_{c} .
$$

Substituting Eq. (6) in Eq. (5) and rearranging it, we get

$R_{S}=\frac{\left(W_{S}-W_{D}\right) \rho}{A}+R_{K}$,

where $R_{K}=R_{n}+R_{a}+R_{c}$

Because of $R_{a}, R_{c}$ and $R_{n}$ characterize resistances out of the depletion layer, their values are independent of the values of first right term of Eq. (7). The resistance $R_{n}$ is n-well dimension dependent, so it has a constant value. Furthermore, the values of both $R_{a}$ and $R_{c}$ may be as possible as minimized during fabrication of the photodiode, as has been mentioned above. Therefore, they may be measured experimentally and then added to the theoretical value of $R_{S}$ as a correction factor. Thus Eq. (7) is reduced to

$R_{S} \approx \frac{\left(100-W_{D}\right) \times 0.075}{A}+R_{K}(\mathrm{Ohm})$.

The dependence of $R_{S}$ on $W_{D}$ for different values of $A$, which is changed from $1 \mathrm{~mm}^{2}$ to $10 \mathrm{~mm}^{2}$ with $R_{K}$ about 5 Ohm, is depicted in Fig. 4.

There are three factors defining the response time of a photodiode: draft time $t_{\text {drift }}$, diffusion time $t_{\text {diff }}$ and time constant $t_{R C}$. The total rise time is determined by:

$\left(t_{r}\right)^{2}=\left(t_{R C}\right)^{2}+\left(t_{\text {drift }}\right)^{2}+\left(t_{\text {diff }}\right)^{2}$,

where the time constant is given as

$t_{R C}=2.2\left(R_{S}+R_{L}\right)\left(C_{D}+C_{S}\right)$,

where $R_{L}$ is the load resistance, and $C_{S}$ is the stray capacitance which can be minimized by using short leads. Thus, Eq. (11) becomes

$t_{R C} \approx 2.2\left(R_{S}+R_{L}\right) C_{D}$.

Substituting Eq. (4) and Eq. (9) in Eq. (12) results

$t_{R C}=0.2274096 \times$

$\times\left[\left(\frac{\left(100-W_{D}\right) \times 0.075}{A}+R_{K}\right)+R_{L}\right] \frac{A}{W_{D}}$.

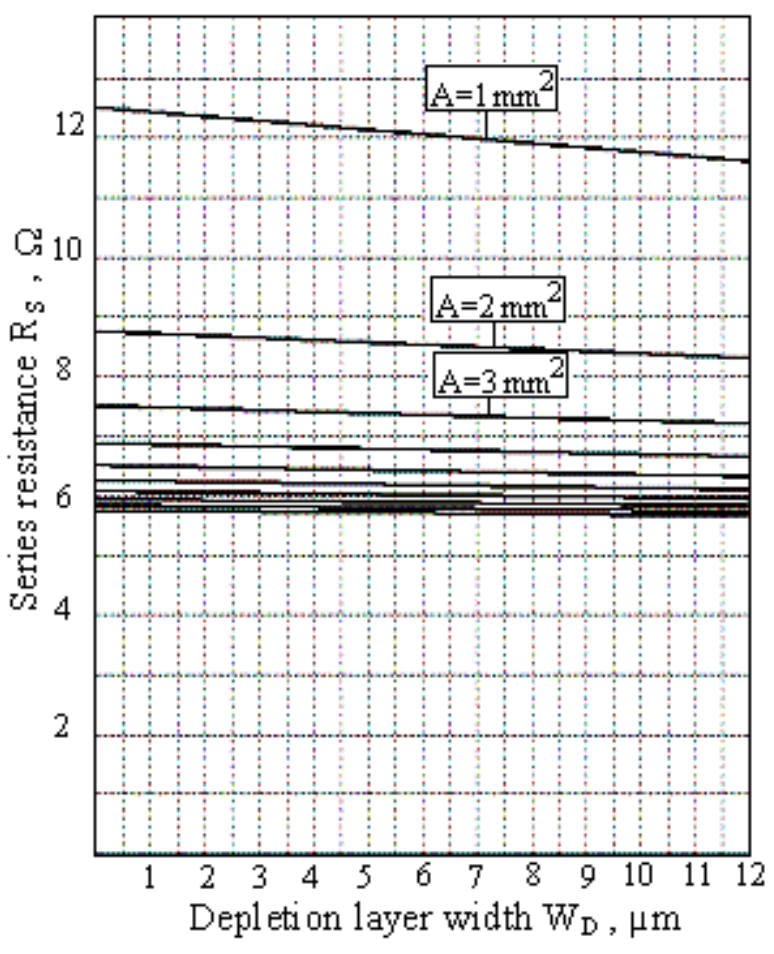

Fig. 4. The dependence of the series resistance on the depletion layer width.

The drift time $t_{\text {drift }}$ for silicon is given as

$t_{\text {drift }}=\frac{W_{D}}{V_{d}}$,

where $V_{d}$ is the average drift velocity of the carriers which is $1 \cdot 10^{7} \mathrm{~cm} / \mathrm{s}$ for silicon at $300 \mathrm{~K}$. By substituting this value in Eq. (14), it becomes

$t_{\text {drift }}=0.01 W_{D}(\mu \mathrm{m})(\mathrm{ns})$.

Figure 5 illustrates the variation of $t_{R C}$ with the depletion layer width for different values of load resistance from 10 to $50 \mathrm{Ohm}$ and the diffused junction area of $5 \mathrm{~mm}^{2}$ as well as the variation of $t_{\text {drift }}$ with the depletion layer width.

Because the research concerns what happens inside the depletion layer, the diffusion time $\left(t_{\text {diff }}\right)$ is out of our study. So, plotting $\left(t_{R C}\right)^{2}+\left(t_{\text {drift }}\right)^{2}$ has just been adopted and shown in Fig. 6.

Figure 7 illustrates superposition of the two graphs of $t_{R C}$ and $t_{\text {drift }}$ to produce the third graph denoted by $\sqrt{\left(t_{R C}\right)^{2}+\left(t_{\text {drift }}\right)^{2}}$ which is the first two terms of $t_{r}$ in Eq. (10).

As shown in Fig. 2 the depletion layer width much decreases with increasing the impurity concentration and decreasing reverse applied potential and vice versa. That is because increasing or decreasing of the concentration of diffusing charge carriers between both sides of the junction. Of course, the narrowest depletion layer causes largest capacitance when the area of the diffused layer is so large, as illustrated in Fig. 3. On the other hand, since 
the substrate thickness is always much larger than the depletion layer thickness, the last one has no longer effect on the series resistance. Therefore, with minimum value of the contacts resistance, the series resistance may be changed few milliohms, as shown in Fig. 4. Thus, the time constant of the photodiode, which is due to series resistance and its total capacitance as well as the load resistance, depends on the depletion layer width. With small value of the load resistance and small diffused junction area, the time constant may be minimized by increasing the depletion layer width due to its effect on the junction capacitance, as has been stated.

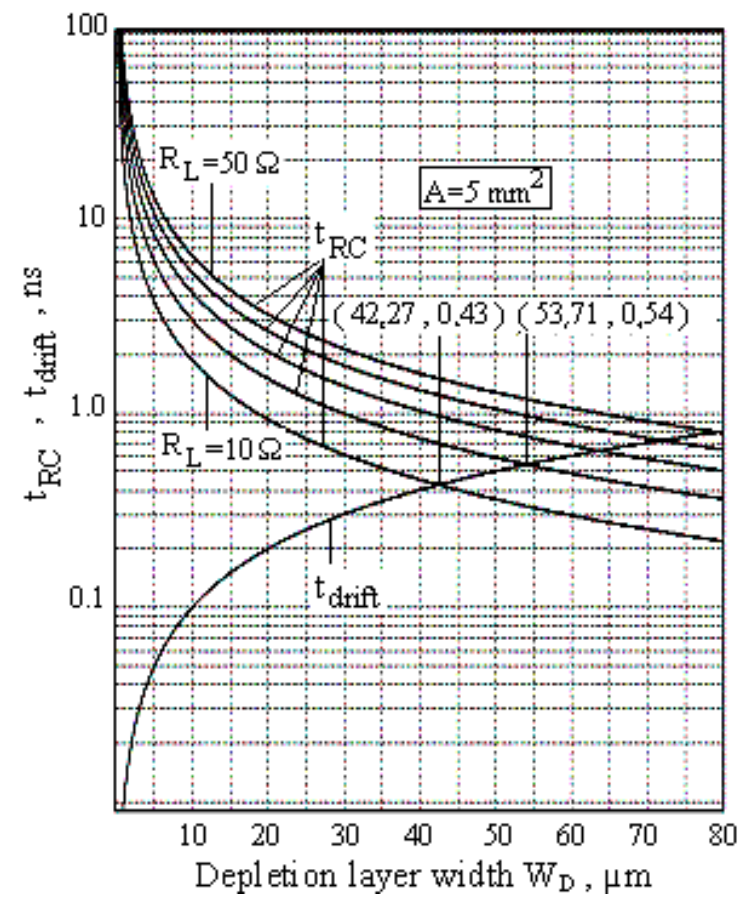

Fig. 5. The variation of $t_{R C}$ and $t_{\text {drift }}$ with the depletion layer width.

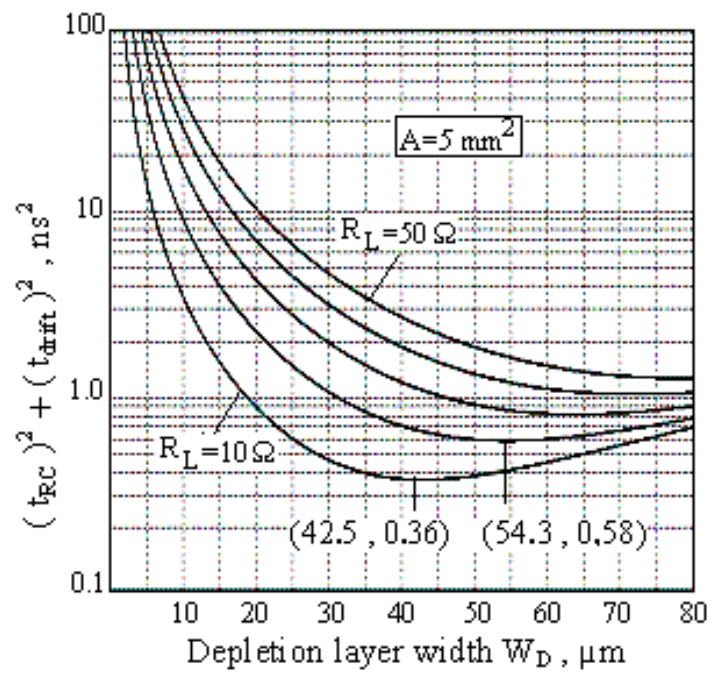

Fig. 6. The variation of $\left(t_{R C}\right)^{2}+\left(t_{\text {drift }}\right)^{2}$ with the depletion layer width.

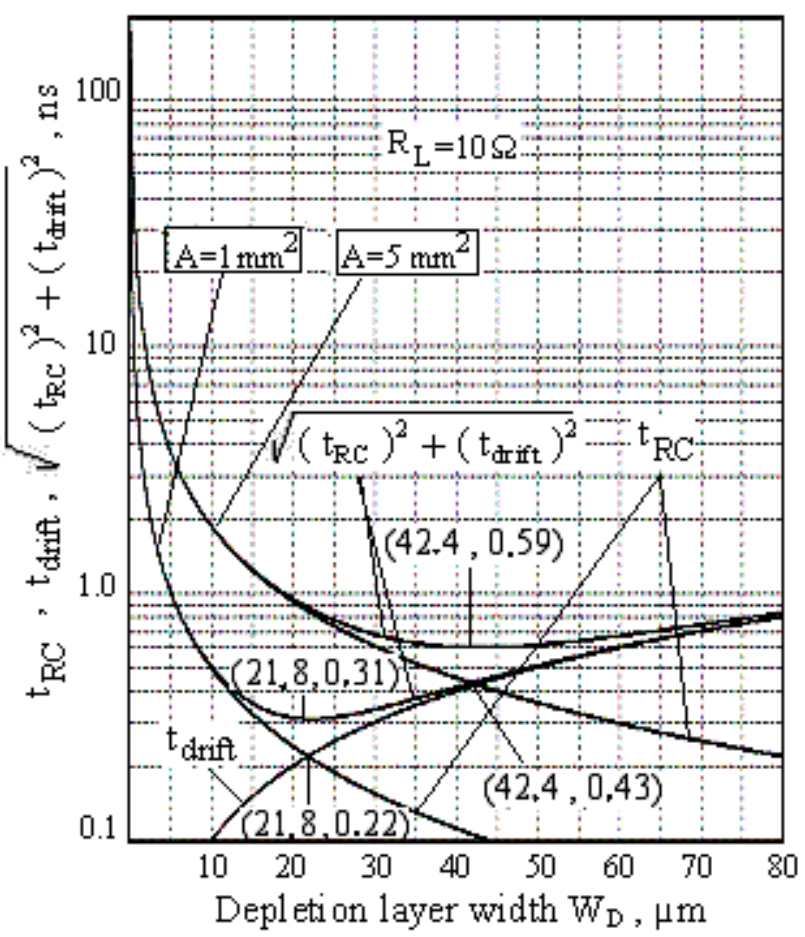

Fig. 7. The variation of $\sqrt{\left(t_{R C}\right)^{2}+\left(t_{\text {drift }}\right)^{2}}$ with the depletion layer width.

In contrast, the drift time increases linearly with the depletion layer width, because of decreasing the electric field within it, and intersects the time constant curves, as illustrated in Fig. 5. So, adding their quadratic values results in the curves shown in Fig. 6 in which the minimum value of each curve lies at the same point of their intersection. That means, the minimum values of them lie at specific values of the depletion layer width.

Returning to Fig. 7, for $R_{L}=10 \mathrm{Ohm}$ and $A=$ $5 \mathrm{~mm}^{2}$, one can see that the plot of the square root of quadratic $t_{R C}$ plus quadratic $t_{\text {drift }}$ is a curve starts from the highest value of $t_{R C}$ corresponding to the minimum value of the depletion layer width and finishes at the highest value of $t_{\text {drift }}$ corresponding to the maximum value of the depletion layer width. Also, its minimum value (0.59 ns) lies at the intersection of both curves, i.e. at $42.4 \mu \mathrm{m}$. Further, for $A=1 \mathrm{~mm}^{2}$ the minimum value becomes $0.31 \mathrm{~ns}$ at depletion layer width of $21.8 \mu \mathrm{m}$.

\section{Conclusions}

Since the junction capacitance of the photodiode is dependent on the depletion layer thickness, the diffused junction area and the applied reverse bias, the minimum value of contribution of both the time constant and the drift time in rise time, are obtained at specific value of the depletion layer width which is corresponding to smaller diffused area, and larger applied reverse bias. Furthermore, for values less than that value of the depletion layer the contribution of the time constant 
dominates the rise time value whereas the contribution of the drift time dominates the rise time for values more than the specific value of the depletion layer. In other words, it is possible to reach an optimal value of the rise time by controlling the depletion layer thickness through the fabrication process of the photodiode.

\section{References}

1. A. Hirata et al., $120-\mathrm{GHz}$ wireless link using photonic techniques for generation, modulation and emission of millimeter-wave signals // J. Lig. Wav. Technol. 21 (10), p. 2145-2153 (2003).

2. G. Eppeldauer, Chopped radiation measurements with large area Si photodiodes // J. Res. Natl. Inst. Stand. Technol. 103 (2), p. 153-162 (1998).

3. A.M. Moloney et al., Monolithically integrated avalanche photodiode and transimpedance amplifier in a hybrid bulk-SOI CMOS process // Elec. Lett. 39 (4), p. 391-392 (2003).

4. S. Malyshev and A. Chizh, High-speed photodiodes for radio-on-fiber communication systems // Proc. sympos. photo. techno. 7th framew. prog., p. 286-290 (2006) (Wroclaw).

5. L. Shi et al., Response time of shallow junction silicon photodiodes // Elect., p. 21-26, Sep. 24-26 (2008) (Bulgaria).

6. R.A. Yotter et al., Optimized CMOS photodetector structures for the detection of green luminescent probes in biological applications // Sen. Act. B 103, p. 43-49 (2004).

7. C.P. Allier et al., Thin photodiode for scintillatorsilicon well detector // IEEE Trans. Nucl. Sci. 46 (6), p. 1948-1951 (1999).

8. T.N. Swe and K.S. Yeo, An accurate photodiode model for DC and high frequency SPICE circuit simulation // Nanotech. 1, p. 362-365 (2001).

9. H.I. Kim et al., Fabrication and characterization of silicon-based photodiode for detection of luminol chemiluminescence in a biosensor // J. Korean Phys. Soc. 42, p. S336-S339 (2003).

10. O. Bazkir and F. Samadov, Characterization of silicon photodiode-based trap detectors and establishment of spectral responsivity scale // Opt. Las. Eng. 43, p. 131-141 (2004).

11. S.H. Lim et al., Photocurrent spectroscopy of optical absorption enhancement in silicon photodiodes via scattering from surface plasma on polaritons in gold nanoparticles // J. App. Phys. 101(104309), (2007).

12. K. Chilukuri et al., Monolithic CMOS-compatible AlGaInP visible LED arrays on silicon on latticeengineered substrates (SOLES) // Semicond. Sci. Technol. 22, p. 29-34 (2007).

13. S.M. Sze and K.K. Ng, p-n junctions, in: Physics of Semiconductor Devices, 3rd ed., Ch. 2, p. 83. John Wiley \& Sons, Inc., New Jersey, 2007. 